exists concerning the actual bonding in organometallic compounds.

$$
\text { G. P. VAN Der Kelen }
$$

Laboratory for General and Inorganic Chemistry, University of Ghent.

${ }^{1}$ Müller, Norbert, and Pritchard, Donald E., J. Chem. Phys., 31, 768 (1959).

2 Shoolery, J. N., J. Chem. Phys., 31, 1427 (1959).

${ }^{3}$ Müller, Norbert, and Pritchard, Donald E., J. Chem. Phys., 31, 1471 (1959).

Bent, H. A., J. Inorg. Nucl. Chem., 19, 43 (1961).

- Burke, John J., and Lauterbur, Paul C., J. Amer. Chem. Soc., 83, 326 (1961).

\section{Ultrasonic Hydroxylation in a Fluorescence Analysis for Microgram Quantities of Benzoic Acid}

ULTRASONIC cavitation is able to bring about a variety of chemical reactions, including oxidation, reduction, polymerization, and depolymerization (see review ${ }^{1}$. Recent emphasis on the role of hydroxyl free radicals produced by ultrasound from water molecules ${ }^{2}$ suggested the possibility of direct ultrasonic hydroxylation of aromatic molecules. (The analogous hydroxylation by means of X-rays has been studied by Weiss et $a l .{ }^{3}$ ).

A use of ultrasonic hydroxylation is described here. It provides a simple new method for the determination of benzoate in pure solution, by measurement of the fluorescence of the hydroxybenzoates produced. This method is sensitive to $1 \mu \mathrm{gm}$. of benzoic acid, compared with $50 \mu \mathrm{gm}$. for either the Nicholls procedure ${ }^{4}$ of hydroxylation by hydrogen peroxide followed by colorimetric determination of the salicylic acid produced, or the Dickens and Pearson method $^{5}$ of nitration, reduction, and diazo coupling.

The new procedure is as follows. Adjust the solution to be not more than $0.00002 M$ in benzoic acid, and make it $0.001 M$ in $p \mathrm{H} 11 \mathrm{Na}_{2} \mathrm{HPO}_{4}-\mathrm{Na}_{3} \mathrm{PO}_{4}$ buffer. Irradiate $20.0 \mathrm{ml}$. in a $32 \times 200 \mathrm{~mm}$. 'Pyrex' test tube for $5.0 \mathrm{~min}$. with ultrasound (about 20-W. power inside the vessel, as determined by a calorimetric substitution method) using a $400-\mathrm{kc} . / \mathrm{s}$. convergentbeam Brush hypersonic generator operated at 160 m.amp. d.c. cathode current. Measure the fluorescence of a sample at $410 \mathrm{~m} \mu$ in an Aminco-Bowman spectrophotofluorimeter, under excitation at $315 \mathrm{~m} \mu$. Carry a $0.00002 M$ standard through the procedure with each set of unknowns, and compute the unknown concentration from the simple ratio of fluorescence intensities. It is necessary to follow a standardized schedule of operations, and to place the test-tube the same way for each irradiation.

Table 1 shows the results obtained (average of duplicate runs); the relative error does not exceed 5 per cent. Interference by equimolar concentrations of acetic or succinic acid is negligible. However, aromatic acids such as cinnamic or phthalic yield interfering fluorescent products.

Attempts were made to increase the induced fluorescence by adding $0.20 \mathrm{ml}$. of 3 per cent hydrogen peroxide to $20 \mathrm{ml}$. of solution before irradiation, by

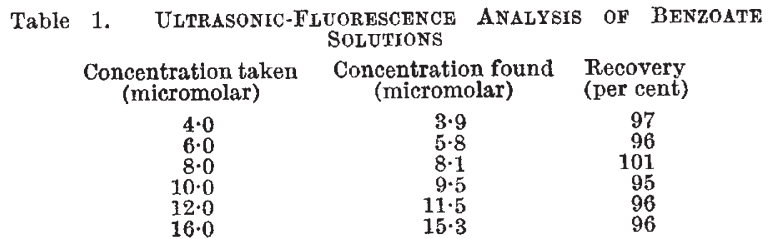

replacing the dissolved air with oxygen in a closed system before irradiation, and by combining both procedures, but in each case the result was negative. The hydroxylation of benzoate in 0.06 per cent hydrogen peroxide solution under the action of the ultraviolet radiation in the spectrophotofluorimeter, but without ultrasound, gave a very slow build-up of the characteristic fluorescence, which amounted after 50 min. to less than half that achieved by $5 \mathrm{~min}$. of ultrasound.

The use of $p \mathrm{H} 11$ buffer gave more intense fluores. cence than in either unbuffered solution $(p \mathbf{H} 6 \cdot 5)$ or $0.001 \mathrm{~N}$ sulphuric acid medium. Under my conditions, slightly more fluorescence was obtained by prolonging the ultrasonic treatment to $10 \mathrm{~min}$., but 15 min. gave a definite decrease, presumably because of partial destruction by the continued action of hydroxyl free radicals.

Nature of products. Analysis of $0.00002 M$ benzoate after $5 \mathrm{~min}$. of ultrasonic irradiation, by the fluorometric method of Thommes and Leininger ${ }^{6}$, indicated conversion into 1 per cent each of salicylate and $m$-hydroxybenzoate. This is consistent (since $p$ hydroxybenzoate is non-fluorescent) with reported results ${ }^{2}$ of the hydroxylation of benzoate by boiling hydrogen peroxide for several hours, yielding at first a few per cent each of the ortho-, meta-, and parahydroxy derivatives, which then undergo further hydroxylation to produce dihydroxybenzoates.

Ultrasonic treatment of $0.01 M$ benzoate for the extended period of $1 \mathrm{hr}$. gave fluorescence emission and excitation spectra which resembled those of gentisic (2,5-dihydroxybenzoic) acid at the same $p H$. Paper chromatography of this solution in $1: 1$ ethanol-water gave four fluorescent spots, of which the brightest by far corresponded to gentisic acid. Two other spots, which were due to $m$-hydroxybenzoic and protocatechuic (3,4-dihydroxybenzoic) acids, were observed even more distinctly by their phosphorescence at $-195^{\circ}$ by pouring liquid nitrogen directly on the paper. A salicylic acid spot was not detected with certainty, although a 'known' spot containing $1 \mu \mathrm{gm}$. in $0.10 \mathrm{ml}$. showed up clearly under the same conditions, possibly because salicylate itself was found to be converted fairly rapidly by ultrasound into gentisate and other products.

Spectrophotometric examination of the irradiated $0.01 M$ benzoate for the presence of phenolic hydroxyls was conducted with the aid of the $\Delta \varepsilon-$ method $^{8}$, in which the spectrum in $2 M$ sodium hydroxide was determined against a blank of the same solution at $p \mathbf{H} 4 \cdot 4$. A strong peak in the $320-330 \mathrm{~m} \mu$ region was found in the irradiated solution, but not in the untreated benzoate. This was very close to the peaks found by the same technique in $m$-hydroxybenzoate, gentisate, and protocatechuate (all of which have an $\mathrm{OH}$ meta to a $\mathrm{COOH}$ group), but distinct from the salicylate peak near $300 \mathrm{mu}$.

Acknowledgment is made of the helpful suggestions of Dr. Robert L. Bowman.

Alfred Weissier

Laboratory of Technical Development, National Heart Institute, Bethesda 14, Maryland.

1. Weissler, A., J. Acoust. Soc. Amer., 25, 651 (1953).

¿ Weissler, A., J. Amer. Chem. Soc., 81, 1077 (1959).

${ }^{3}$ Loebl, H., Stein, G., and Weiss, J., J. Chem. Soc., 405 (1951).

${ }_{4}^{4}$ Nicholls, J. R., Analyst, 53, 19 (1928).

'Dickens, F., and Pearson, J., Biochem. J., 48, 216 (1951)

- Thommes, G. A, and Leininger, E., Anal, Chem., 30, 1361 (1958).

"Dakin, H. D., and Herter, M. D., J. Biol. Chem., 3, 419 (1907).

A Aulin-Erdtman, G., Chem. and Indust., 581 (1955). 